\title{
SCOPING REVIEW ON PRODUCTIVITY-RELATED PSYCHOLOGICAL INTERVENTIONS: INTEGRATION INTO THE FELICITY APP
}

\author{
Dec 5, 2020
}

Joy Xu, ${ }^{1}$ Jeffrey Ong, ${ }^{1}$ Muntaha Marjia, ${ }^{2}$ Aaron Lio, ${ }^{3}$ Harshdeep Dhaliwal ${ }^{1}$

${ }^{1}$ McMaster University, Hamilton, ON, Canada

${ }^{2}$ Ryerson University, Toronto, ON, Canada

${ }^{3}$ St Robert CHS, Thornhill, ON, Canada

\begin{abstract}
Background

The Felicity App is a mobile application undergoing development with the aim to integrate practical psychological interventions with recreational elements to reinforce positive and productive behaviours. Prior to construction, psychological interventions must be reviewed for relevance, effectiveness and ability to be integrated within Felicity. Psychological concepts are reviewed as the preliminary phase prior to further investigation in subfields.
\end{abstract}

\section{Methods}

Electronic databases were searched including (and not limited to) PsychInfo, PubMed, Web of Science, and ScienceDirect from November 29, 2020 to December 5, 2020. Further filtering methods were applied accordingly, and grey literature was excluded. Common and effective psychological interventions related to productivity were screened and extracted, with an emphasis on the reinforcement and discouragement of productivity-related behaviours.

\section{Results}

Productivity-related psychological interventions were addressed according to three categories: 1) Motivation 2) Procrastination 3) Time management. Within motivation, self-affirmation is an effective method, particularly when accompanied with other positive thinking techniques. Intrinsic motivation could be enhanced with goals aligned in specific criterias. Additionally, virtual rewards, engagement, and familiarity serve as vital components to enhance motivation. Procrastination is associated with lower engagement in work and interventions include various therapy measures. Within time management, clear purpose has been shown as an effective tool for goal setting while strategies include spaced learning and other study techniques.

\section{Conclusion}

This scoping review determined several points of connection between motivation, procrastination, and time management with many effective interventions which could be potentially integrated within the Felicity App for personal development and habit-building. Additional research should be conducted to further determine the effectiveness of interventions for integration into Felicity.

\section{BACKGROUND}

The Felicity App is a mobile application created to enhance productivity in a recreational and practical way. Integrated with effective psychological interventions, this app aims to incorporate positive reinforcements for educational/work productive behaviours. The application centralizes around user input for goal orientation and setting. Features will include productivity tools such as to-do lists, calendars, and reminder notifications. Positive reinforcement tools include psychological interventions like mental health 
exercises (such as self-affirmations, mindfulness, and other techniques), time management tips (delivered through notifications and avatar narratives), and rewards for productive behaviours (through point-systems and potential leaderboards). Prior to the construction of the Felicity App, the research department of the app development team will undergo thorough investigation for effective psychological interventions with input from experts of the field and conduct detailed studies. This study serves as an overview of potential psychological concepts to be integrated within the Felicity App.

\section{METHODS: PSYCHOLOGICAL CONCEPTS FOR INTEGRATION}

\section{Overview of Research Integration Within the Felicity App}

The Felicity App is currently in preliminary stages of development as technological frames and scientific-based evidence undergo in-depth investigation. The research department of the application development team plans to navigate effective psychological interventions with an advisory board of mental health professionals across North America. Mental health professionals (including and not limited to psychiatrists, $\mathrm{PhD} / \mathrm{MDs}$, therapists, and others) will be scoped based on academic merit within psychological fields related to motivation, cognitive processes, productive behaviours, and learning. Upon acceptance into the advisory board, research will be conducted and critically appraised in several phases throughout the upcoming six months.

An initial interview procedure will take place to determine logistical factors, including availability and other aspects with selected advisors. With consideration to the technological department of the app development team, appraisal sessions will be held on a maximal interval of one-hour discussions every two weeks, excluding other online communications such as emails. Advisors will be presented with updates on research and potential game element integrations by research department subgroups. Appraisal sessions will be held with one to three research department members and one to two advisors to ensure independent evaluation and discussion. Advisors will assess validity, reliability, and other elements of research presented by the team members with predetermined criteria. Additionally, advisors will discuss personal areas of expertise with potential integration into the application.

To ensure in-depth exploration, the research department will be subdivided into three groups for centralized areas of focus including motivation, procrastination, and time management. Within these groups, team members may undergo independent studies for detailed exploration. To minimize potential bias or errors, each group will independently determine interventions for inclusion, with group evaluation on a monthly basis and advisor appraisal on a biweekly basis. Advisors will not be provided compensation, with exception to an end-of-project honorarium, as a means to minimize potential competing interests.

Through critical appraisal, finalized research concepts and interventions will accompany recreational elements to maximize user experience and encourage positive and productive behaviour.

\section{Search Method}

Prior to determining specific areas of focus, this study aims to highlight potential psychological concepts for further investigation within motivation, procrastination, and time management. Potential psychological interventions will address concepts of productivity in young adults, particularly in students and young employees. Online databases and journals were searched independently from November 29, 
2020 to December 5, 2020. The following inclusion criteria was utilized to guide the search while reviewing articles:

$>$ Published in the English Language

$>$ Human subjects

$>$ Published from $1995-2020$

$>$ Age groups from 14 to 65 years old

$>$ All gender/race inclusive

$>$ Targets general population or young adult population in developed countries

Study exclusion pertained to populations with specific diseases/illnesses, grey literature, or if studies did not adequately follow the inclusion criteria. The following electronic databases were included and not limited to PsychInfo, PubMed, Web of Science, and ScienceDirect. Search keywords were used including "procrastin*", "motivat*", "procrastination intervent*", "time management methods", "college", "learning", "grades", "procrastination prevention", "cognitive therap*”, "positive affect", "affirm*”, "strateg*" and others. Filtering methods were applied according to the inclusion criteria in addition to operational functions (AND or OR) to narrow results depending on the area of focus.

\section{RESULTS}

\section{Motivation}

\section{Self-Affirmation Theory}

Self-affirmation theory promotes motivation through maintaining positive perceptions of one's competence and identity (Sherman \& Cohen, 2006, 183). Affirmation exercises have been shown as effective measures for consolidation of one's core values, with neural mechanisms showing significant effects within the brain's reward system (Cascio et al., 2015, 627). Hence, reinforcement learning through self-affirmation shows biological support, accompanied by correlated measured behaviour change to suggest external validity of affirmation (Cascio et al., 2015, 628).

Additionally, positive affirmation may foster intrinsic motivation and promote responsible behaviour for goal orientation and completion (Isen \& Reeve, 2005, 298). In a study conducted by Isen et al., intrinsic motivation was found to be fostered by positive affect for both enjoyable and uninteresting tasks alike (Isen \& Reeve, 2005, 300). Subsequently, a relationship is demonstrated between affirmation and positive affect for effective cognitive processes. Affirmatory statements are most effective when accompanied with other positive thinking techniques and goal-setting methods (Harackiewicz \& Elliot, 1993, 906). Once goals have been defined, reciting, reading, or hearing these affirmations several times a day, and most importantly, before high pressure situations, can assist in sustaining intrinsic motivation (Harackiewicz \& Elliot, 1993, 912).

\section{Goal Setting Theory of Motivation}

In 1990, researchers Edwin Locke and Gary Latham analyzed nearly 400 studies related to goal development and task performance (Lunenburg, 2011, 2). Through this appraisal, Locke and Latham developed the Goal Setting Theory of Motivation, seen in Figure 1. 


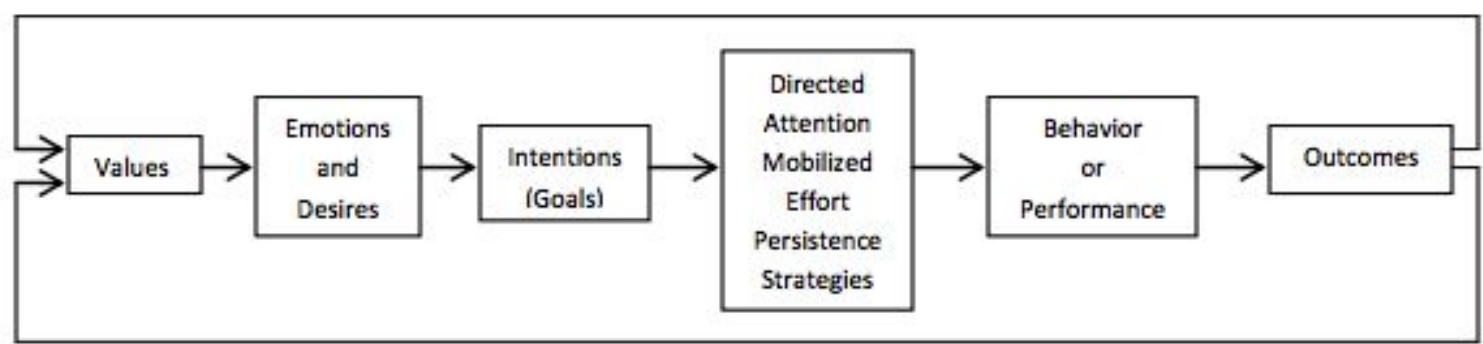

Frustration and Lower Motivation

Figure 1. General model of goal-setting theory. Values and intentions (goals) were two cognitive determinants of behaviour, while one's values elicit desire with tasks corresponding to similar values (Lunenburg, 2011, 2).

While goal-setting serves as an effective technique of increasing motivation, maximal performance could be attained through the following criteria:

1. Specificity: Goals must outline specific elements, including what to reach for, how to measure progress, and the reward upon completion (Lunenburg, 2011, 2).

2. Challenging: Goals must be difficult but realistically attainable (Lunenburg, 2011, 3).

3. Acceptable: Goal-setters should create organic goals, rather than being assigned to goals (Lunenburg, 2011, 3).

4. Feedback: Upon attainment, feedback must be provided to the goal-setter (Lunenburg, 2011, 3).

5. Evaluation: Goals are more effective when performance is assessed. Hence, academic goals have greater impact when directly linked to marks (in addition to student learning) (Lunenburg, 2011, 4).

6. Deadlines: Set due dates improve the effectiveness of goals, partially attributed to increasing specificity of established goals (Lunenburg, 2011, 4).

7. Learning oriented: Goals should be learning-oriented, rather than performance oriented (Lunenburg, 2011, 4).

\section{Gamification Theory}

Gamification theory reinforces intrinsic motivation within individuals by providing virtual rewards (such as badges and avatars) to successful game players (Kamasheva et al., 2015, 77). Three distinct game mechanics include the olympiad, win-win, and aesthetic mechanics (Kamasheva et al., 2015, 78). Socialist competitions have been shown as a useful tool to enhance relationships amongst individuals in an academic or workplace setting, resulting in greater productivity as individuals feel intrinsically inclined to communicate amongst themselves (Kamasheva et al., 2015, 79).

\section{Positive Psychology in Students}

An absence or lack of motivation can be sourced to several factors, including disengagement, anxiety, failure avoidance, self-handicapping, and uncertain control (Martin, 2005, 116). Conversely, eleven 
factors serving as motivation reinforcers include planning, study management, self-efficacy, mastering orientation, valuing of school, and persistence (Martin, 2005, 116). Therefore, targeting and recognizing these areas of focus could enable individuals to overcome periods of procrastination and gain motivation to complete one's tasks.

\section{The Influence of Familiarity}

Tine Heafner discussed the role of familiarity which enables students to be more willing when engaging in tasks which utilize familiar approaches/resources (Heafner, 2004, 47). Specifically, the effectiveness of familiarity has been observed as students displayed greater self-efficacy and confidence within their cognitive abilities when using technology to complete a task, as the students had more fluency towards technology, thereby increasing their level of motivation (Heafner, 2004, 44).

\section{Role of Motivation in Web-Based Tools}

Web-based motivational tools may not have a facilitator, but in a standard classroom scenario, facilitators can promote interaction and humanize instruction, thus increasing motivation (Martin, 1997, 93). In combination to familiar and practical resources, students thrive with engaging elements including the ability to question, present, and discuss (as opposed to passive means of listening to lectures or taking notes from textbooks) (Martin, 1997, 201).

\section{Keller's Motivation-Design Model}

The Keller's Motivation-Design Model is a method for designing motivational tools with four implemented categories; interest (eliciting curiosity), relevance (linking learning/work to the needs/motives of the worker), expectancy (likelihood they will re-approach a similar task), and satisfaction (continuing motivation- desire to continue pursuing goals) (Martin, 1997, 96).

In reference to extrinsic awards, the gamification of the learning process can increase psychological need satisfaction (Sailer et al., 2017, 371). Gamification utilized game design elements for non-gaming purposes in real world settings to promote human performance for specific tasks (Sailer et al., 2017, 371). Previous research has found a correlation which supports this aim, where the following elements were investigated in non-game contexts: (1) points, (2) badges, (3) leaderboards, (4) performance graphs, (5) meaningful stories, (6) avatars and (7) teammates (Sailer et al., 2017, 373). Each of these elements will be assessed and embedded into Felicity as appropriate with further investigation.

\section{Procrastination (Prevention Strategies)}

\section{Work Engagement:}

Work engagement refers to an enthusiastic, positive, and fulfilling mindset at work (Hoppe et al., 2018, 185). The alleviation of procrastination has been correlated with empowering (rather than imposing) tasks where one feels in control of influencing set goals and may participate avidly (Hoppe et al., 2018, 187). Additionally, setting written expectations provides one to internally align performance goals through feelings of self-control rather than domination (Hoppe et al., 2018, 193). Though imposed tasks do not necessarily relate to procrastination, there is a negative relation to engagement which may reduce one's feelings of control over tasks (Hoppe et al., 2018, 193). Thereby, a perception of high autonomy and supportive approach is negatively associated with procrastination, seen in student-teacher relationships (Codina et al., 2020, 2449). 
In work environments where employees enjoy completing their assigned tasks, the probability of their active engagement in irrelevant activities such as viewing videos on social media is decreased (Metin et al., 2018, 229). Therefore, if employers make changes to the work environment of their employees in an effort to increase work engagement, they may see less workplace procrastination. Such changes may include offering employees an incentive (such as providing bonuses to retail employees with the greatest number of sales by the end of a certain time period) to encourage workers to be more engaged and dedicated to their jobs (Metin et al., 2018, 231).

\section{Common Forms of Intervention for Self Regulation}

Self regulation integrates management of internal (i.e. attention, emotion, motivation) and external (i.e. environment, support, time) factors (Eerdea \& Klingsieck, 2018, 78). Enhancement of these properties include self-reflection, regulation of emotion, and stimulus control (Eerdea \& Klingsieck, 2018, 78). Common forms of interventions include:

$>$ Cognitive-behaviour therapy (CBT) focuses on identifying functionality of thoughts as means of transforming behaviour (Eerdea \& Klingsieck, 2018, 78).

$>$ Coherence therapy aims at greater understanding of emotional roots in certain symptoms (Eerdea \& Klingsieck, 2018, 78).

$>$ Acceptance-based behaviour therapy encourages one to view negative emotions as transient and natural while defining behaviour-directing experiences and instill positive thinking patterns (Eerdea \& Klingsieck, 2018, 78).

Upon intervention, procrastination tendencies decrease with medium to large decline, suggesting the significance of self-regulation as part of goal fulfillment (Eerdea \& Klingsieck, 2018, 81). Follow-up measurements indicate stable effects upon procrastination intervention, with little return of procrastination tendencies (Eerdea \& Klingsieck, 2018, 82).

CBT has been shown with larger effect sizes, potentially due to intensity of the interventions (Eerdea \& Klingsieck, 2018, 82). Though procrastination is viewed as a failure of self-regulation by many studies, CBT's effectiveness may be explained by its encouragement of one's initiative.

\section{Reality Therapy Based Training Programs}

Reality therapy helps individuals understand and accept that they are responsible for the consequences of their choices (Çelik \& Odacı, 2017, 222). Counselors specializing in this form of psychotherapy help individuals make decisions that may direct their lives in a way that leads to desired outcomes.

Psycho-educational training sessions that are based upon reality therapy concepts have been proven to be an effective way to reduce academic procrastination behaviour (Çelik \& Odac1, 2017, 228). They teach students skills that can help them reduce their procrastination and improve their academic performances. The skills are related to a variety of different areas including shifting one's mindset, time management, developing a positive outlook of oneself, and learning strategies (Çelik \& Odacı, 2017, 222).

\section{Time Management}

\section{Time Management and Academic Stress}

Throughout the world, there has been an upward trend in student stress, potentially attributed by the increase in expectation for students to gain extensive knowledge within shorter periods of time (Häfner \& 
Stock, 2010, 431). Excessive stress serves as a determinant to potential physical and psychological impairment. Hence, time management strategies can help students regain a sense of control over their time and reduce negative emotions and behaviours.

\section{Time Management Behaviours}

Individuals who perform more time management behaviours have greater self efficacy, higher levels of morale and lower levels of distress. Hafner \& Stock (2009) proposed a model of four behaviours arranged in a hierarchy of importance. At the top, the most important behaviour is having a clear purpose, where those with an ultimate goal in mind are more likely to feel effective at work (Kearns \& Gardiner, 2007, 242). In addition, having a clear sense of purpose appears to boost morale and decrease levels of distress. At the next level of importance is planning and prioritizing (Kearns \& Gardiner, 2007, 242). Those who plan their time and prioritize their tasks not only feel more effective at work, but also have higher morale (Kearns \& Gardiner, 2007, 242). The final two behaviours, avoiding distractions and being organized, were not found to be significant in improving perceived effectiveness, but may provide some buffering effect against distress while doing work (Kearns \& Gardiner, 2007, 243).

\section{Spaced Learning}

Repetitive training and elaboration enhances the formation of long-term memory (Smolen et al., 2016, 77). Spaced learning is characterized by learning with long intervals between each session, and has been shown to have greater effectiveness in inducing memory formation (Smolen et al., 2016, 77). One proposed theory of the mechanism behind the superiority of spaced training is the study-phase retrieval theory. This theory posits that spaced stimulus presentations or learning trials are more effective than massed trials for memory reinforcement as each spaced trial elicits retrieval and reactivation of a memory trace that was formed by the preceding trial (Smolen et al., 2016, 78). By contrast, with short massed trials, the preceding memory trace is still active, so it is not retrieved or reactivated and therefore the memory cannot be reinforced (Smolen et al., 2016, 78).

\section{To-Do Lists}

It has been demonstrated that having a written plan of action increases productivity (Schrager \& Sadowski, 2016, 10). The act of planning activities through "to-do" lists reduces executive burden on the brain by freeing the brain from having to worry about unfinished tasks. This is called the Zeigarnik Effect, named after psychology Bluma Zeigarnik (Schrager \& Sadowski, 2016, 10). The recommended approach involves prioritizing to-do lists into urgent and not urgent, important and not important (Schrager \& Sadowski, 2016, 10). Breaking down a task into smaller tasks can also help make the to-do list more effective (Schrager \& Sadowski, 2016, 12)

\section{Multitasking}

Multitasking is a misnomer as the human brain truly focuses on one task at a time, where tasks are switched back and forth when one multitasks (Schrager \& Sadowski, 2016, 12). Researchers also documented a cognitive "bottleneck" at a central area of information processing, which allowed only 1 thought through at a time (Schrager \& Sadowski, 2016, 12). Other research has shown that it takes 30-60 seconds to refocus on 1 task after transferring attention to a second one (Schrager \& Sadowski, 2016, 10). The more complex the task, the longer it takes to refocus, hence, breaking down a complex task into smaller tasks can help one stay focused for longer periods of time (Schrager \& Sadowski, 2016, 12). 


\section{Taking breaks: Ties to the Pomodoro Technique}

In sensory perception, a phenomenon known as habituation occurs when the brain's response to a repeated stimulus diminishes over time (Ariga \& Lleras, 2011, 440). When attempting to maintain a focused attention for prolonged periods of time, a similar phenomenon known as vigilance decrement occurs (Ariga \& Lleras, 2011, 442). Myriad studies have shown that performance on tasks often show a downsloping curve as a function of time. Ariga \& Lleras (2011) proposed a new account of vigilance decrement known as goal habituation, stating that the cognitive control system may have difficulties in maintaining a goal active for a prolonged period of time. Results from their study show that temporarily deactivating the vigilance goal would prevent full goal habituation from occurring by re-strengthening the goal's activation level upon resumption of the task (Ariga \& Lleras, 2011, 443). With that in mind, taking frequent breaks during long study sessions may be beneficial for improving overall performance and productivity.

\section{DISCUSSION}

\section{Motivation}

With reference to the aforementioned elements of motivation, each factor can be integrated into the layout, function, or appearance of the app. Goal-setting elements will be prominent; including the ability for users to set goals, receive feedback on their goals, and introspectively reflect on the effectiveness and achievability of their goals. Moreover, the app will be populated with integrated psychological interventions including affirmative statements and encouragement, narratives from the avatar and the notification system. With reference to the gamification of Felicity, user dashboards will be able to visualize figures including "time spent working" and other performance markers to allow the app to improve their learning experience. Other potential factors would include leaderboard functions, graphs for time spent studying among friends, ability for users to add friends, and badges/points for productivity achievements. This will allow individuals to stimulate intrinsic motivation and would aid in the development of habits for long-term motivation. As Felicity is an online platform, users will have an initial sense of familiarity upon accessing it, which could be reinforced with a simplistic and user-friendly layout. Since users are more likely to engage in technology that they are familiar with, commonly used social media aspects could be considered for incorporation into Felicity as well.

With respect to affirmation statements within Felicity, a diagnostic survey for users can be developed to help users recognize potential source(s) for their lack of motivation, using the categories established by the student motivation and engagement wheel. Thereby, users can check-in on one's mental state, while also practicing strategies to promote motivation, as Felicity will provide strategies to prevent or reduce the effect of the demotivating sources, after the users complete the survey. Overall, Felicity's main focus is to help its users stay productive through maintaining mental health, which can be reinforced by in-game rewards, as mentioned above, along with attendance check-ins.

\section{Procrastination}

The application can provide many opportunities for employees and students to increase their work engagement. Felicity can enable individuals to feel like they are more in control of their goals. This may be done by having a section within the application where individuals can create lists of their required 
tasks. There may also be an area within this section in which users can provide an explanation for why a task is important and the impact it may have when it is finished. Use of this feature can allow individuals to reflect on their goals and understand why they exist which may increase their likelihood of completing their tasks efficiently with reduced procrastination. Another way Felicity can improve work engagement is by making completing tasks more enjoyable for users. This could be done by offering an incentive such as unlocking special features within the app, if the user completes a certain amount of tasks that are worth a certain number of points. Also, perhaps the application could be used in conjunction with a music application. Depending on the task and the music preferences of the user, Felicity may suggest playlists or songs that could be played as the person completes their task (for example, LoFi music if the user is having a studying session). Overall, when people enjoy doing their work, their engagement is increased which may reduce their procrastination.

Felicity will potentially integrate the strategies used in various interventional therapies including cognitive-behaviour therapy, coherence-therapy, acceptance-based therapies, and reality therapy to help users reduce their procrastination tendencies. For example, a section could be created within the app where users can reflect on their productivity. In their reflections, individuals may discuss why certain time management-strategies did not work, why they think they were feeling less productive and ways in which they can improve. Through the reflections, users may reflect on the consequences of their choices which have led to procrastination (for example, if an individual delayed starting a project, they now have less time to complete it and must work faster which may lead to the end product not meeting their expectations). Furthermore, more positive thinking patterns should be encouraged within users and show them not to feel overly disappointed when they cannot finish all of their tasks on a certain day as an acceptable outcome. Overall, the purpose of this aspect of the application is to encourage users to view negative experiences as an opportunity to learn and become more productive while confronting potential problems accompanied with procrastination habits.

\section{Time Management}

The integration of time management strategies into the functions of Felicity can assist users to maintain high levels of productivity and performance. When users first enter the application to input their tasks, users will be suggested to write out a main goal or task that they wish to accomplish that day. Having a clear purpose helps the user focus and direct their efforts towards completing that task or goal. Moreover, on the main screen, the application could have a section to input tasks and rank them in terms of urgency. This links back to the time management behaviour of "Planning and Prioritizing". Making a to-do list helps users break down tasks into smaller, more manageable chunks, which can prevent the user from feeling overwhelmed. Having smaller tasks also demotes multitasking as the user is able to focus for longer periods of time on a single task. Finally, making a to-do list takes advantage of the Zeigarnik Effect. By planning out the day, users are less likely to be interrupted by anxiety-triggering thoughts about unfinished tasks.

Having a clear goal, planning and prioritizing are important in deciding what users want to accomplish, but may not necessarily affect their method of working. Felicity can help by integrating a productivity system into its functions. The potential implemented system may mirror the Pomodoro technique for time management as a system where the user works on a task for 25 minutes, then takes a 5 minute break. This completes one cycle. Once four cycles have been completed, the user can then take a longer 30 minute 
break. Within the application, there's potential for a timer capable of setting specific intervals of working and resting times. Taking frequent breaks prevents goal habituation from occurring, and helps users stay focused on a single task until the timer goes off. The Pomodoro technique also helps prevent users from burning out by having the extended break every four cycles.

The final component is repetitive learning. Within Felicity, features may be integrated with the to-do list where users can decide if they want to revisit a specific task in the future, and set a date for when they wish to work on the task again. This feature would benefit students the most as the majority of assessments in educational institutions are based around memorizing key concepts. Having repetitive learning integrated into the application makes it easy for users to effectively plan out a study schedule and stay organized.

\section{Conclusion}

The Felicity App aims to encourage positive mindset and behaviour by targeting potential causal factors to procrastination while enhancing time management and motivation. Through integrating various psychological concepts and interventions, this mobile application offers an incentivizing and impactful solution to self-improvement which could be applied to any user interested in developing productive habits. This scoping review provides an overview of key areas relevant to the Felicity App. These results enable greater insight towards the app development process and serve to highlight potential fields for further investigation. Quality appraisal and statistical analyzes were not performed due to the scoping review nature. However, additional comprehensive research will be required to develop potential realms of psychological interventions to be integrated into Felicity.

\section{Contributions}

All authors have made substantive intellectual contributions to developing this scoping review. All authors report no competing interests and this research project was not funded. 


\section{References}

Ariga, A., \& Lleras, A. (2011). Brief and rare mental "breaks" keep you focused: Deactivation and reactivation of task goals preempt vigilance decrements. Cognition, 118(3), 439-443. https://doi.org/10.1016/j.cognition.2010.12.007

Cascio, C. N., O’Donnell, M. B., Tinney, F. J., Lieberman, M. D., Taylor, S. E., Strecher, V. J., \& Falk, E. B. (2015). Self-affirmation activates brain systems associated with self-related processing and reward and is reinforced by future orientation. Social Cognitive and Affective Neuroscience, 11(4), 621-629. https://doi.org/10.1093/scan/nsv136

Çelik, Ç. B., \& Odacı, H. (2017). Psycho-Educational Group Intervention Based on Reality Therapy to Cope with Academic Procrastination. Journal of Rational-Emotive \& Cognitive-Behavior Therapy volume, 36, 220-233.

https://doi.org/10.1007/s10942-017-0283-1

Codina, J., Castillo, I., Pestana, J. V., \& Balaguer, I. (2020). Preventing Procrastination Behaviours: Teaching Styles and Competence in University Students. Sustainability, Motivation and the Teaching-Learning Process in Physical Education and Sport, 12(6), 2448-2459. https://doi.org/10.3390/su12062448

Eerdea, W. v., \& Klingsieck, K. B. (2018). Overcoming procrastination? A meta-analysis of intervention studies. Educational Research Review, 25, 73-85. https://doi.org/10.1016/j.edurev.2018.09.002

Häfner, A., \& Stock, A. (2010). Time Management Training and Perceived Control of Time at Work. Full Terms \& Conditions of access and use can be found at https://www.tandfonline.com/action/journalInformation?journalCode $=$ vjrl20 The Journal of Psychology, 144(5), 429-447. https://doi.org/10.1080/00223980.2010.496647 
Harackiewicz, J. M., \& Elliot, A. J. (1993). Achievement goals and intrinsic motivation. Journal of Personality and Social Psychology, 65(5), 904-915. https://doi.org/10.1037/0022-3514.65.5.904

Heafner, T. (2004). Using Technology to Motivate Students to Learn Social Studies. Contemporary Issues in Technology and Teacher Education, 4(1), 42-53. https://citejournal.org/volume-4/issue-1-04/social-studies/using-technology-to-motivate-s tudents-to-learn-social-studies

Hoppe, J. D., Prokop, P., \& Rau, R. (2018). Empower, not impose!-Preventing academic procrastination. Journal of Prevention \& Intervention in the Community, 46(2), 184-198. https://doi.org/10.1080/10852352.2016.1198172

Isen, A. M., \& Reeve, J. (2005). The Influence of Positive Affect on Intrinsic and Extrinsic Motivation: Facilitating Enjoyment of Play, Responsible Work Behavior, and Self-Control. Motivation and Emotion, 29, 295-323. https://doi.org/10.1007/s11031-006-9019-8

Kamasheva, A. V., Valeev, E. R., Yagudin, R. K., \& Maksimova, K. R. (2015). Usage of Gamification Theory for Increase Motivation of Employees. Mediterranean Journal of Social Sciences, 6(1), 77-80. https://doi.org/10.5901/mjss.2015.v6n1s3p77

Kearns, H., \& Gardiner, M. (2007). Is it time well spent? The relationship between time management behaviours, perceived effectiveness and work- related morale and distress in a university context. Higher Education Research \& Development, 26(2), 235-247. https://doi.org/10.1080/07294360701310839

Lunenburg, F. C. (2011). Goal-Setting Theory of Motivation. INTERNATIONAL JOURNAL OF MANAGEMENT, BUSINESS, AND ADMINISTRATION, 15(1), 1-6. 
http://www.nationalforum.com/Electronic\%20Journal\%20Volumes/Lunenburg,\%20Fred \%20C.\%20Goal-Setting\%20Theoryof\%20Motivation\%20IJMBA\%20V15\%20N1\%2020 11.pdf

Martin, A. J. (2005). The Role of Positive Psychology in Enhancing Satisfaction, Motivation, and Productivity in the Workplace. Journal of Organizational Behavior Management, 24(1-2), 113-133. https://doi.org/10.1300/J075v24n01_07

Martin, B. L. (1997). Web-based Instruction. Language Arts \& Disciplines.

Metin, U. B., Peters, M. C.W., \& Taris, T. W. (2018). Correlates of procrastination and performance at work: The role of having "good fit". Journal of Prevention \& Intervention in the Community, 46(3), 228-244. https://doi.org/10.1080/10852352.2018.1470187

Sailer, M., Hanseb, J. U., Mayr, S. K., \& Mandla, H. (2017). How gamification motivates: An experimental study of the effects of specific game design elements on psychological need satisfaction. Computers in Human Behaviour, 69, 371-380.

https://doi.org/10.1016/j.chb.2016.12.033

Schrager, S., \& Sadowski, E. (2016). Getting More Done: Strategies to Increase Scholarly Productivity. Journal of Graduate Medical Education, 8(1), 10-13. http://dx.doi.org/10.4300/JGME-D-15-00165.1

Sherman, D. K., \& Cohen, G. L. (2006). The Psychology of Self-defense: Self-Affirmation Theory. Advances in Experimental Social Psychology, 38, 183-242. https://doi.org/10.1016/S0065-2601(06)38004-5

Smolen, P., Zhang, Y., \& Byrne, J. H. (2016). The right time to learn: mechanisms and optimization of spaced learning. Nature Reviews Neuroscience, 17(2), 77-88. https://doi.org/10.1038/nrn.2015.18 